\title{
THE CARDINALITY OF QUASICONFORMALLY NONEQUIVALENT TOPOLOGICAL 3-BALLS WITH FLAT BOUNDARIES IS c
}

\begin{abstract}
RAIMO NÄKKI
Abstract. The theorem mentioned in the title is proved. During the course of the proof, the failure for $n=3$ of the following 2-dimensional result will also be established: The boundary of a Jordan domain $D$ in $n$-space is a quasiconformal $(n-1)$-sphere if every quasiconformal self-mapping of $D$ can be extended to a quasiconformal self-mapping of the whole space.
\end{abstract}

1. Introduction. Let $\Sigma^{n-1}$ be a topological $(n-1)$-sphere imbedded in the $n$-sphere $S^{n}$. By the Jordan-Brouwer separation theorem, $\Sigma^{n-1}$ divides $S^{n}$ into two domains, $D_{1}$ and $D_{2}$, and is their common boundary. The set $\Sigma^{n-1}$ is collared if there is a neighborhood $U$ of $\Sigma^{n-1}$ and a homeomorphism $h$ of $U \cap \bar{D}_{1}$ (or of $U \cap \bar{D}_{2}$ ) into $S^{n}$ carrying $\Sigma^{n-1}$ onto the equator $S^{n-1}$ of $S^{n}$. The set $\Sigma^{n-1}$ is bicollared if $h$ is defined in all of $U$ and maps $\Sigma^{n-1}$ onto $S^{n-1}$. The set $\Sigma^{n-1}$ is flat if there is a homeomorphism $h$ of $S^{n}$ onto itself which carries $\Sigma^{n-1}$ onto $S^{n-1}$. By results of Brown [1], [2], every bicollared $\Sigma^{n-1}$ in $S^{n}$ is flat and a collared component of $S^{n}-\Sigma^{n-1}$ is a topological $n$-ball.

The set $\Sigma^{n-1}$ is said to be quasiconformally collared (resp. quasiconformally bicollared) if the homeomorphism $h$ above is quasiconformal. The image of $S^{n-1}$ under a quasiconformal mapping of $S^{\mathrm{n}}$ is generally referred to as a quasiconformal sphere, rather than a quasiconformally flat sphere. Gehring [4] has established quasiconformal analogues to Brown's results. In particular, a quasiconformally collared component of $S^{n}-\Sigma^{n-1}$ is a quasiconformal $n$-ball. The other component of $S^{n}-\Sigma^{n-1}$ need not be a quasiconformal $n$-ball, even in the case that $\Sigma^{n-1}$ is flat.

Let $\mathscr{D}$ be the collection of all topological $n$-balls in $S^{\mathrm{n}}$ whose boundaries are flat $(n-1)$-spheres and whose exteriors are quasiconformal $n$-balls. We divide $\mathscr{D}$ into equivalence classes by regarding two domains in $\mathscr{D}$ as equivalent if they can be mapped quasiconformally onto each other. Let $E(D)$ denote the set of equivalence classes so obtained. We show that in 3-space $E(D)$ has the cardinality of a continuum. This stands in marked contrast with the situation in 2-space, where the corresponding cardinality is well known to be one. (For related questions, see Kopylov [6].) In the course of the proof, the failure for $n=3$ of the following result, due to Rickman [8]

Received by the editors February 20, 1978.

AMS (MOS) subject classifications (1970). Primary 30A60, 30A24, 30A30; Secondary 54A25.

Key words and phrases. Cardinality, quasiconformal mapping, modulus of a curve family, topological ball, quasiconformal sphere, flat boundary. 
for $n=2$, will also be established: The boundary of a Jordan domain $D$ in $n$-space is a quasiconformal $(n-1)$-sphere if every quasiconformal selfmapping of $D$ can be extended to a quasiconformal self-mapping of the whole space.

2. Wedges. We consider domains $D$ in $\bar{R}^{3}=R^{3} \cup\{\infty\}$,

$$
D=\left\{x=\left(x_{1}, x_{2}, x_{3}\right) \in R^{3}:\left|x_{2}\right|<g\left(x_{1}\right), x_{1}>0\right\},
$$

where the function $g:[0, \infty) \rightarrow R^{1}$ satisfies the following conditions for some $0<a<\infty$ :

$$
\left\{\begin{array}{l}
\text { (i) } \quad g \text { is continuous, } g(0)=0, g(u)>0 \text { for } u>0, \\
\text { and } g(u)=g(a) \text { for } u \geqslant a . \\
\text { (ii) } g^{\prime} \text { is continuous, bounded, and increasing in } \\
\\
(0, a) . \\
\text { (iii) } \lim _{u \rightarrow 0} g^{\prime}(u)=0 .
\end{array}\right.
$$

Such a domain $D$ is called a wedge of angle zero. The union of the $x_{3}$-axis and the point $\infty$ is called the edge of $D$. (The above terminology is taken from Gehring and Väisälä [5].) Obviously a wedge $D$ is a Jordan domain whose boundary $\partial D$ is a flat 2-sphere. The exterior of $D$ is a quasiconformal 3-ball, while $D$ is not. (See Gehring and Väisälä [5].) Hence $\partial D$ is not a quasiconformal 2-sphere, i.e. $\partial D$ is not quasiconformally bicollared.

We will show that no two of the wedges defined by the functions $g(u)=$ $u^{p}, p \in(1, \infty)$, can be mapped quasiconformally onto one another. For this we require an upper and a lower bound for the modulus $M(\Gamma)$ of a certain path family $\Gamma$. We let $F(\Gamma)$ denote the set of all Borel-measurable extended real-valued functions $\rho$ in $R^{3}$ for which

$$
\int_{\gamma} \rho d s \geqslant 1
$$

for each locally rectifiable path $\gamma \in \Gamma$. The modulus of $\Gamma$ is defined as

$$
M(\Gamma)=\inf _{\rho \in F(\Gamma)} \int_{R^{3}} \rho^{3} d m .
$$

(For the theory of modulus and quasiconformal mappings, see Gehring [3] and Väisälä [10].)

LemMa 1. Let $D$ be the wedge defined by the function $g(u)=u^{p}(p>1)$, let $r_{0}>0$ be a number such that $0<g^{\prime}\left(r_{0}\right) \leqslant 1$, for $0<r \leqslant r_{0}$ let

$$
Z(r)=\left\{x=\left(x_{1}, x_{2}, x_{3}\right): x_{1}^{2}+x_{3}^{2}<r\right\},
$$

and for $0<r_{1}<r_{2} \leqslant r_{0}$ let $\Gamma\left(r_{1}, r_{2}\right)$ denote the family of all paths joining $\partial Z\left(r_{1}\right)$ and $\partial Z\left(r_{2}\right)$ in $D \cap Z\left(r_{2}\right)-\overline{Z\left(r_{1}\right)}$. Then 


$$
\frac{A(p)}{\left(r_{1}^{(1-p) / 2}-r_{2}^{(1-p) / 2}\right)^{2}} \leqslant M\left(\Gamma\left(r_{1}, r_{2}\right)\right) \leqslant \frac{2^{3 / 2} A(p)}{\left(r_{1}^{(1-p) / 2}-r_{2}^{(1-p) / 2}\right)^{2}},
$$

where

$$
A(p)=2^{-5 / 2}(p-1)^{2} \int_{0}^{\pi}(\sin \varphi)^{p} d \varphi
$$

Proof. For the left-hand inequality, let $\rho \in F\left(\Gamma\left(r_{1}, r_{2}\right)\right)$, let $\left(r, \varphi, x_{2}\right)$ be cylindrical coordinates in $R^{3}$ with the polar angle $\varphi$ being measured from the positive half of the $x_{3}$-axis, and for $r \in\left[r_{1}, r_{2}\right], \varphi \in(0, \pi), v \in(-1,1)$ let

$$
\gamma_{\varphi v}(r)=(r, \varphi, v g(r \sin \varphi)) \text {. }
$$

Since $\gamma_{\varphi v}$ is a rectifiable path in $\Gamma\left(r_{1}, r_{2}\right)$ and since $g^{\prime}\left(r_{2}\right) \leqslant 1$ by hypothesis and by (2), we obtain

$$
\begin{aligned}
1 & \leqslant\left(\int_{\gamma_{\varphi v}} \rho d s\right)^{3} \leqslant\left(2^{1 / 2} \int_{r_{1}}^{r_{2}} \rho d r\right)^{3} \\
& \leqslant 2^{3 / 2} \int_{r_{1}}^{r_{2}} \rho^{3} r g(r \sin \varphi) d r\left(\int_{r_{1}}^{r_{2}} r^{-1 / 2} g(r \sin \varphi)^{-1 / 2} d r\right)^{2}
\end{aligned}
$$

by Hölder's inequality. Integrating with respect to $\varphi$ and $v$ yields

$$
\begin{aligned}
\int_{R^{3}} \rho^{3} d m & \geqslant \int_{-1}^{1} d v \int_{0}^{\pi} d \varphi \int_{r_{1}}^{r_{2}} \rho^{3} r g(r \sin \varphi) d r \\
& \geqslant A(p)\left(r_{1}^{(1-p) / 2}-r_{2}^{(1-p) / 2}\right)^{-2},
\end{aligned}
$$

where $A(p)$ is as in (3). Since $\rho \in F\left(\Gamma\left(r_{1}, r_{2}\right)\right)$ was arbitrary, this gives the left-hand inequality.

The right-hand inequality is obtained by observing that

$$
\rho(x)=\left\{\begin{array}{l}
\frac{p-1}{2 r^{(p+1) / 2}\left(r_{1}^{(1-p) / 2}-r_{2}^{(1-p) / 2}\right)} \\
\quad \text { if } x=\left(r, \varphi, x_{2}\right) \in D \cap Z\left(r_{2}\right)-\overline{Z\left(r_{1}\right)}, \\
0 \text { otherwise, }
\end{array}\right.
$$

belongs to $F\left(\Gamma\left(r_{1}, r_{2}\right)\right)$.

We also need the following extension result which shows, in particular, that, contrary to the situation in the plane (Rickman [8]), the extendability of 3-dimensional quasiconformal mappings over a flat 2-sphere does not guarantee that the 2-sphere will be quasiconformal.

LEMMA 2. All quasiconformal mappings between wedges can be extended to quasiconformal self-mappings of $\bar{R}^{3}$.

Proof. This result was proved in [7]. 
LEMMA 3. Let $D$ and $D^{*}$ be two wedges defined respectively by the functions $g(u)=u^{p}$ and $g^{*}(u)=u^{p^{*}}, p, p^{*} \in(1, \infty)$. Then $D$ can be mapped quasiconformally onto $D^{*}$ if and only if $p=p^{*}$.

Proof. The sufficiency part is obvious. For the necessity part, suppose, for example, that $p<p^{*}$, and that, contrary to the assertion, there is a quasiconformal mapping $f$ of $D$ onto $D^{*}$. By Lemma $2, f$ can be extended to a quasiconformal mapping of $\bar{R}^{3}$ onto itself. Denote this mapping again by $f$, let $E$ denote the common edge of $D$ and $D^{*}$, and for $x \in E-\{\infty, f(\infty)\}$ set

$$
L\left(x, f^{-1}\right)=\limsup _{h \rightarrow 0} \frac{\left|f^{-1}(x+h)-f^{-1}(x)\right|}{|h|} .
$$

In the proof of Lemma 2 it is verified that $f(E)=E$. Utilizing an idea of Syčev [9], we note the existence of a point $x_{0}$ in $E-\{\infty, f(\infty)\}$ such that

$$
L\left(x_{0}, f^{-1}\right)>0 \text {. }
$$

Otherwise $f^{-1}$ would be locally constant in $E-\{\infty, f(\infty)\}$. Assume, for convenience of notation, that $x_{0}=0=f^{-1}\left(x_{0}\right)$. Let

$$
\begin{gathered}
L(r)=\max _{|x|=r}|f(x)|, \quad l(r)=\min _{|x|=r}|f(x)|, \\
H=\limsup _{r \rightarrow 0} \frac{L(r)}{l(r)} .
\end{gathered}
$$

Since $H<\infty$ by the quasiconformality of $f$, there exist positive constants $r_{0}$ and $H_{0}$ with $g^{\prime}\left(r_{0}\right) \in(0,1]$ such that

$$
L(r) / l(r) \leqslant H_{0}
$$

whenever $r \in\left(0, r_{0}\right]$. Choose $r_{0}^{*}$ so that $g^{* \prime}\left(r_{0}^{*}\right) \in(0,1]$ and $D^{*} \cap Z\left(r_{0}^{*}\right)$ lies in $f\left(D \cap Z\left(r_{0}\right)\right)$, where, for $r \in(0,1], Z(r)$ is as defined in Lemma 1 . Next choose $c \in\left(0, L\left(0, f^{-1}\right)\right)$ and let $\left(x_{k}\right)$ be a sequence of points in $D$ such that $x_{k} \rightarrow 0,\left|x_{k}\right|<r_{0},\left|f\left(x_{k}\right)\right|<r_{0}^{*}$, and

$$
\left|x_{k}\right| /\left|f\left(x_{k}\right)\right| \geqslant c .
$$

Denoting $\left|x_{k}\right|=r_{k}$ and using (4) and (5) we obtain

$$
L\left(r_{k}\right) \leqslant H_{0} l\left(r_{k}\right) \leqslant H_{0}\left|f\left(x_{k}\right)\right| \leqslant H_{0} r_{k} / c=C_{0} r_{k},
$$

where $C_{0}=H_{0} / c$. Passing to a subsequence, we may assume that $C_{0} r_{k}<r_{0}^{*}$ for every $k$. Since $g^{\prime}\left(r_{0}\right) \leqslant 1$, it follows from (6) that $f\left(D \cap Z\left(r_{k} / 2\right)\right)$ lies in $D^{*} \cap Z\left(C_{0} r_{k}\right)$. Let $\Gamma\left(r_{k} / 2, r_{0}\right)$ be the family of all paths joining $\partial Z\left(r_{k} / 2\right)$ and $\partial Z\left(r_{0}\right)$ in $D \cap Z\left(r_{0}\right)-\overline{Z\left(r_{k} / 2\right)}$ and let $\Gamma^{*}\left(C_{0} r_{k}, r_{0}^{*}\right)$ be the family of all paths 
joining $\partial Z\left(C_{0} r_{k}\right)$ and $\partial Z\left(r_{0}^{*}\right)$ in $D^{*} \cap Z\left(r_{0}\right)-\overline{Z\left(C_{0} r_{k}\right)}$. Since $f \Gamma\left(r_{k} / 2, r_{0}\right)$ is minorized by $\Gamma^{*}\left(C_{0} r_{k}, r_{0}^{*}\right)$, we obtain

$$
\begin{aligned}
\frac{M\left(\Gamma\left(r_{k} / 2, r_{0}\right)\right)}{M\left(f \Gamma\left(r_{k} / 2, r_{0}\right)\right)} & \geqslant \frac{M\left(\Gamma\left(r_{k} / 2, r_{0}\right)\right)}{M\left(\Gamma^{*}\left(C_{0} r_{k}, r_{0}^{*}\right)\right)} \\
& \geqslant 2^{-3 / 2} \frac{A(p)\left[\left(r_{k} / 2\right)^{(1-p) / 2}-r_{0}^{(1-p) / 2}\right]^{-2}}{A(p)\left[\left(C_{0} r_{k}\right)^{\left(1-p^{*}\right) / 2}-r_{0}^{*\left(1-p^{*}\right) / 2}\right]^{-2}} \\
& \geqslant C^{*} r_{k}^{p-p^{*}}
\end{aligned}
$$

by Lemma 1 , where $C^{*}$ is a positive constant which does not depend on $k$. Letting $k \rightarrow \infty$ leads to a contradiction with the quasiconformality of $f$. The proof is complete.

3. Results. Since the boundary of a wedge is not a quasiconformal sphere, Lemma 2 yields:

THEOREM 1. In 3-space there are Jordan domains $D$ whose boundaries are flat, but not quasiconformally flat, such that all quasiconformal self-mappings of $D$ can be extended to quasiconformal self-mappings of $\bar{R}^{3}$.

Since the cardinality of the collection of all subdomains of $\bar{R}^{3}$ is c, the cardinality of a continuum, Lemma 3 yields:

THEOREM 2. In 3-space the cardinality of a maximal collection of quasiconformally nonequivalent Jordan domains with flat boundaries and quasiconformally collared exteriors is $\mathbf{c}$.

\section{REFERENCES}

1. M. Brown, A proof of the generalized Schoenflies theorem, Bull. Amer. Math. Soc. 66 (1960), 74-76.

2. , Locally flat imbeddings of topological manifolds, Ann. of Math. (2) 75 (1962), $331-341$.

3. F. W. Gehring, Rings and quasiconformal mappings in space, Trans. Amer. Math. Soc. 103 (1962), 353-393.

4. Extension theorems for quasiconformal mappings in n-space, J. Analyse Math. 19 (1967), 149-169.

5. F. W. Gehring and J. Väisälä, The coefficients of quasiconformality of domains in space, Acta Math. 114 (1965), 1-70.

6. A. P. Kopylov, On the richness of the class of quasiconformal mappings of domains in three-dimensional Euclidean space, Dokl. Akad. Nauk SSSR 172 (1967), 527-528. (Russian)

7. R. Näkki, Extension of quasiconformal mappings between wedges, Proceedings of the Romanian-Finnish seminar on Teichmüller spaces and quasiconformal mappings, Brašov, 1971, pp. 229-233.

8. S. Rickman, Extension over quasiconformally equivalent curves, Ann. Acad. Sci. Fenn. AI 436 (1969), 1-12. 
9. A. V. Syčev, Quasiconformal mappings in space, Dokl. Akad. Nauk SSSR 166 (1966), 298-300. (Russian)

10. J. Väisälä, Lectures on n-dimensional quasiconformal mappings, Lecture Notes in Math., vol. 229, Springer-Verlag, Berlin and New York, 1971.

Department of Mathematics, University of Helsinka, Helsinka, Finland

Department of Mathematics, University of Michigan, ANn Arbor, Michigan 48109

Current address: Department of Mathematics, University of Texas, Austin, Texas 78712 